\title{
EVOLUÇÃO DA MORTALIDADE POR CÂNCER CÉRVICO-UTERINO EM SALVADOR - BA, 1979-1997*
}

\author{
Susanne Andrade Derossi* \\ Jairnilson Silva Paim* \\ Estela Aquino* \\ Lígia Maria Vieira da Silva*
}

\begin{abstract}
RESUMO: O câncer cérvico-uterino, quando diagnosticado e tratado precocemente, constitui-se em uma causa de morte perfeitamente evitável. Entretanto, no Brasil, a mortalidade por esta causa ainda é elevada, persistindo como problema de saúde pública. O objetivo deste trabalho é descrever a evolução da mortalidade e estimar os anos potenciais e produtivos de vida perdidos por esta neoplasia, em Salvador-Bahia, entre 1979 e 1997. Trata-se de um estudo de agregados de série temporal, no qual foram considerados todos os óbitos de mulheres com idade igual ou superior a 20 anos, que tiveram como causa básica de morte o câncer de colo uterino e de porção não especificada do útero. Utilizou-se como fontes de dados o CD ROM: DATA SUS/ MS, o IBGE e a Secretaria de Saúde do Estado da Bahia/SESAB. Os indicadores empregados foram as taxas de mortalidade específicas por idade, brutas e padronizadas por idade. Houve um decréscimo de $50,6 \%$ na taxa padronizada de mortalidade por este tipo de câncer, no período analisado, cujos valores variaram de 17,6/100.000 mulheres em 1979 a 8,7 em 1997. Observou-se ainda que o risco de morte por esta causa cresce à medida que aumenta a idade e que a sua magnitude torna-se mais expressiva a partir de 40 anos. A variação da média de anos potenciais de vida perdidos por mulher foi de 15,5 no ano de 1986 a 20,4 em 1980. Os autores concluem que a mortalidade por câncer cérvico-uterino neste município ainda é alta e discutem os possíveis fatores determinantes da evolução desta mortalidade. Destacam a necessidade de ações efetivas na prevenção da morbi-mortalidade por esta causa.
\end{abstract}

PALAVRAS-CHAVE: câncer cérvico-uterino; mortalidade; tendência; neoplasia.

* Instituto de Saúde Coletiva da Universidade Federal da Bahia - UFBA. 


\section{INTRODUÇÃO}

O câncer cérvico-uterino é uma doença de evolução gradativa, que se inicia com alterações neoplásicas intra-epiteliais, as quais podem evoluir para um processo invasivo em um período médio de 10 a 20 anos. Como possui etapas bem definidas e evolução lenta, permite a interrupção do seu curso a partir de um diagnóstico precoce e tratamento oportuno (OPS, 1985).

Em países com programas organizados de screening com adequada cobertura, as taxas de incidência e de mortalidade por este câncer vêm se reduzindo (ROBLES, 1996). Desse modo, esse tipo de neoplasia está sob controle nos países industrializados, o que não ocorre naqueles de desenvolvimento dependente, onde persiste como problema de saúde pública (BRASIL, 1996).

No Brasil, foi estimada para região Sudeste, em 1999, a taxa de incidência do câncer cervical, de 18,2/100.000 mulheres, enquanto o valor deste indicador correspondeu a 43,9 no Norte, e a 33,1 no Nordeste, ou seja, 2,4 e 1,8 vezes maior do que naquela região, respectivamente, revelando desigualdades regionais na ocorrência desta doença (BRASIL, 1999).

Apesar das características que the conferem um melhor prognóstico, o câncer cervical invalida e mata anualmente milhares de mulheres no Brasil, face às coberturas ineficientes dos programas de prevenção deste agravo, por parte das Secretarias de Saúde (BRASIL, 1994). Neste país, em 1990, a mortalidade por esta causa, ajustada por idade, segundo população padrão mundial, foi de 5,5/100.000 mulheres, ou seja, quase quatro vezes superior à do Canadá e dos Estados Unidos, onde estas taxas foram de 1,4 e 1,7, respectivamente (ROBLES, 1996). Esta neoplasia foi responsável pela morte de 4.663 mulheres no país, em 1988, o que o tornou a sétima causa de morte por câncer na população geral. Sendo de 6.815, o número absoluto de óbitos estimado para 1998, significa um crescimento de $46,2 \%$ nestes dez anos, representando a segunda causa de óbito por câncer entre mulheres no Brasil e $15 \%$ dos tumores malignos do sexo feminino (BRASIL, 1991,1994,1998). Neste mesmo ano, o Ministério da Saúde realizou uma Campanha Nacional de Combate ao Câncer de Colo Uterino, tendo como objetivo a redução da morbimortalidade por esta doença, através da realização do teste Papanicolaou em aproximadamente 3.600.000 mulheres, prioritariamente na faixa de 35-49 anos. Embora tenha definido como uma de suas metas iniciar o tratamento de todas as mulheres com qualquer indício de infecção, lesão ou câncer no colo do útero, seis meses após o final da campanha não havia informações disponíveis sobre a situação das mulheres que apresentaram alterações em seus exames (BRASIL, 1999). 
Em Salvador, capital do estado da Bahia, a mortalidade proporcional por câncer de útero (colo de útero, útero e porção não especificada) em relação às principais localizações de neoplasias malignas em mulheres adultas, no ano de 1995 , foi de $14,3 \%$, e também ocupou a segunda posição em relação a todos os óbitos por câncer entre mulheres (BRASIL, 1998). No Hospital Aristides Maltez, especializado em câncer neste município, o carcinoma de colo do útero correspondeu a 30,8\% dos tumores mais freqüentes, no ano de 1993 (BRASIL, 1994).

A relevância da neoplasia cervical como determinante de óbito entre mulheres nesse município, tanto pela sua magnitude, como por se tratar de uma causa de morte evitável, aliado à carência de estudos mais detalhados sobre a mortalidade por esta causa, determinaram o interesse desta pesquisa. Assim, este trabalho tem como objetivo descrever a evolução da mortalidade por câncer cérvico-uterino e estimar seu impacto em anos potenciais e anos produtivos de vida perdidos na cidade de Salvador, no período compreendido entre 1979 e 1997.

\section{METODOLOGIA}

Trata-se de um estudo de agregados de série temporal, realizado em Salvador, que em 1996 possuía 2.211.539 habitantes, dos quais 1.172.017 eram do sexo feminino.

Considerou-se todos os óbitos de mulheres residentes no referido município, com idade igual ou superior a 20 anos, que faleceram no período de 1979 e 1997, e que tiveram como causa básica de morte o câncer de colo uterino e de porção não especificada do útero. Estas causas correspondem, respectivamente, aos códigos 180 e 179 na 9ª Revisão da Classificação Internacional de Doenças/CID (utilizadas na seleção das causas básicas dos óbitos ocorridos entre 1979 e 1995) e a C53.8, C53.9 e C55, na CID/10ª Revisão (empregada na codificação dos óbitos ocorridos nos anos de 1996 e 1997). A decisão de se incluir os cânceres de porção não especificada do útero deveu-se ao fato de que estudos realizados em São Paulo apontaram que $85 \%$ deles referiam-se a câncer de colo de útero (PUFFER apud MENDONÇA, 1993).

A fonte de dados para os óbitos ocorridos de 1979 a 1995 foi o CD ROM: DATA SUS produzido pelo Ministério da Saúde, e para os anos de 1996 e 1997 foram consultados relatórios fornecidos pelo Centro de Informações de Saúde, da Secretaria de Saúde do Estado da Bahia (CIS/SESAB). 
Para cada ano do estudo, foram calculadas as taxas de mortalidade específicas por idade e as taxas brutas, sendo estas padronizadas pelo método direto, usando-se como referência a População Padrão Mundial (ROUQUAYROL, 1994). As estimativas da população, utilizadas como denominadores para o cálculo desses indicadores, foram fornecidas pelo CIS/SESAB, exceto as populações dos anos de 1980, 1991 e 1996, cuja fonte foi o IBGE.

A análise dos dados foi feita através da representação gráfica da curva de tendência temporal das taxas brutas e padronizadas de mortalidade, bem como pela sua comparação com outra curva delineada a partir do emprego da técnica estatística das médias móveis integradas auto-regressivas de ordem 5 (SPIEGEL, 1985).

Para o cálculo dos Anos Potenciais de Vida Perdidos/APVP foi utilizada a população feminina da faixa etária de 20-69 anos, em sub-grupos de dez em dez anos (LAURENTI et al., 1985). Para a estimativa dos anos produtivos de vida perdidos, considerou-se como 60 anos, o limite determinado para o cálculo.

O processamento dos dados foi feito eletronicamente, com a utilização do Microsoft Excel versão 8.0, e os gráficos foram elaborados pelo emprego do programa Microsoft Grafh 97.

\section{RESULTADOS}

No período analisado, a taxa de mortalidade por câncer cérvico-uterino declinou de 20,7/100.000 mulheres em 1979 para 10,6 em 1997, o que representou uma redução de $48,8 \%$. Esta tendência manteve-se após a padronização desses indicadores, com a ampliação do decréscimo para 50,6\%, entretanto este declínio não foi uniforme, observando-se picos destas taxas nos anos de 1982, 1988 e 1990 (Anexo 1 e Gráfico 1). 

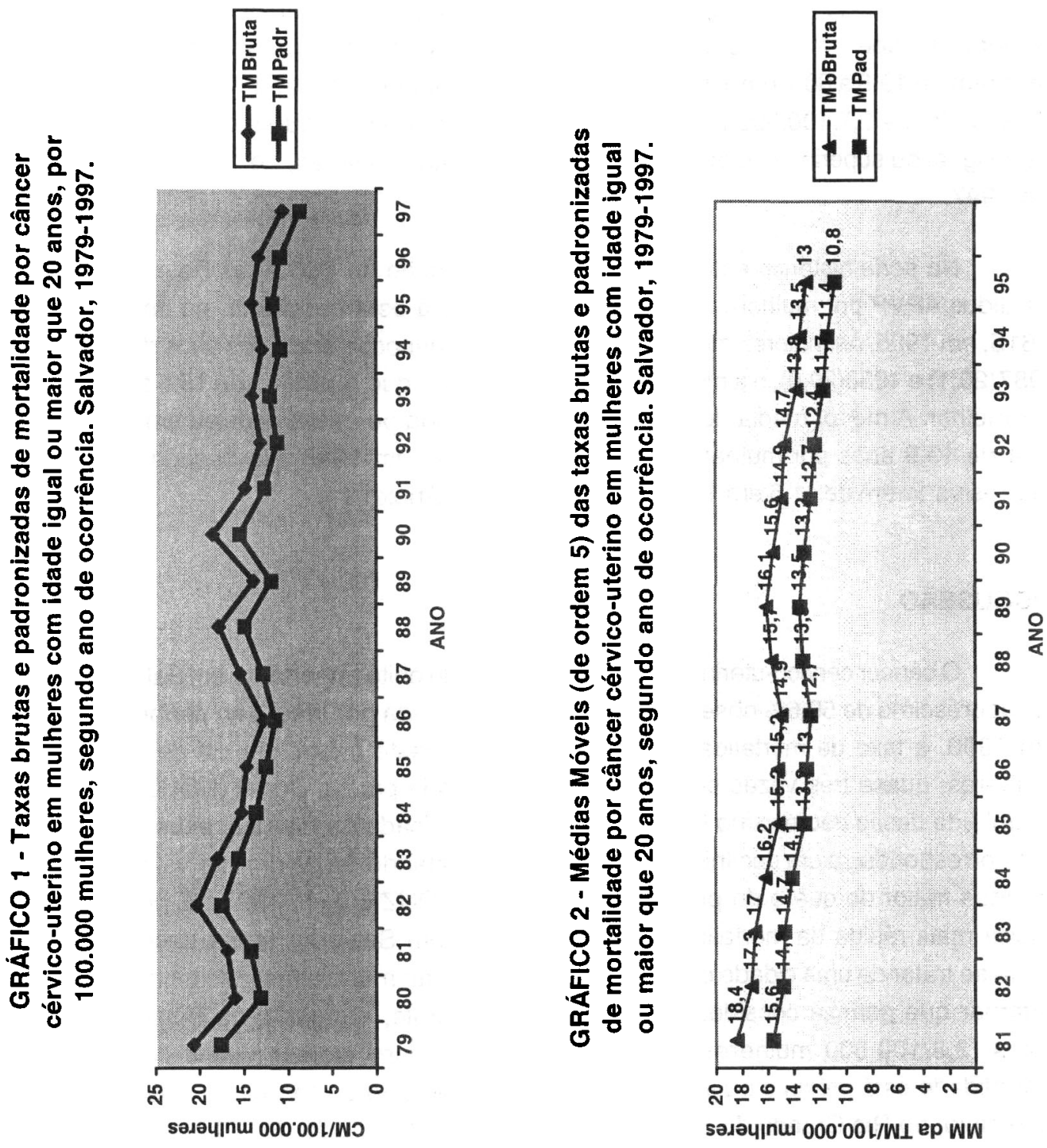
Observa-se no anexo 1, que o risco de morte por esta causa cresce à medida que aumenta a idade e mais acentuadamente a partir dos 40 anos, quando atingiu valores que variaram de 19,5 a 60,7 em 1996, por exemplo. A maior taxa de mortalidade na faixa de 2029 anos foi de 2,7/100.000 mulheres, no ano de 1995. Verificou-se que para aquelas de idade igual ou superior a 70 anos, houve uma variação das taxas, de 137,6 em 1979 a 54,3 em 1997.

Na série histórica estudada houve uma variação de 680 Anos Potenciais de Vida Perdidos/APVP por mulheres que faleceram devido a esta neoplasia, no ano de 1986, a 1.615, em 1990. As maiores médias de APVP por mulher ocorreram nos anos de 1980(20,4), $1987(20,1)$ e 1988(20,0), e a menor ocorreu em 1986, o que representou 15,5 anos perdidos por mulher. A menor média de anos produtivos de vida perdidos ocorreu em 1986, sendo esta de 10,9 anos por mulher, e a maior foi de 15,4, em 1988. Na década de 90 , estes valores variaram de 11,6 em 1997, a 14,3 em 1995 (Anexo 2 ).

\section{DISCUSSÃO}

O câncer cérvico-uterino ainda é uma causa de óbito importante em Salvador, apesar do decréscimo de $50,6 \%$ observado na taxa padronizada por idade, no período analisado. Em 1990, a taxa de mortalidade por esse agravo, neste município, foi de 15,5/100.000 mulheres, quase três vezes superior àquela verificada para o Brasil (ROBLES, 1996). A magnitude desse decréscimo foi superior àquela verificada na Bélgica, entre 1955 e 1989, que correspondeu a um declínio de 39,7\%, mesmo tratando-se de um intervalo de tempo de 15 anos maior do que o do presente estudo (VYSLOUZILOVA, 1997). É possível que a queda mais rápida da mortalidade por esta causa em Salvador tenha decorrido do fato desta se tratar de uma cidade em que as taxas ainda são muito altas, não tendo atingido um patamar que permita considerar a mesma sob controle, enquanto na Bélgica as baixas taxas (3,8/100.000 mulheres em 1985-1988) parecem indicar que o controle desta mortalidade está próximo ou já foi alcançado. Ao contrário da tendência observada neste município, no Rio Grande do Sul, esta mortalidade , no período de 1970 a 1989, mostrou ascendente, com a taxa padronizada de 7,37/100.000 mulheres em 1989, e em São Paulo este valor correspondeu a 4,0, com um leve decréscimo entre 1980 (4,82/100.000 mulheres) e 1989, enquanto em Salvador, o valor deste indicador, neste mesmo ano, foi de 11,9. A tendência observada no Rio Grande do Sul foi atribuída, dentre outros fatores, a uma possível melhoria da acuidade diagnóstica e/ou cuidado no preenchimento dos atestados de óbito (DIETZ, 1993; FONSECA, 1992). 
FAERSTEIN (1987) indicou que o subregistro de óbito acontece principalmente nas camadas mais pobres da população, por razões sócio-econômicas, onde o câncer cervical é mais freqüente. Certamente, o subregistro e sub-notificação de óbitos são fatores que devem ser considerados na análise do presente trabalho, pois os mesmos podem ter influenciado na magnitude da mortalidade em Salvador, porém, se for assumido que não houve alteração no sistema de informação de morte neste período, pode-se acreditar que a tendência ao declínio observada, seja uma realidade. Esta hipótese pode se constituir em um futuro objeto de investigação.

Possivelmente, um outro fator determinante da evolução desta mortalidade em Salvador é a informação da população acerca da importância do cuidado com a saúde, cujo acesso foi facilitado através da mídia, com a modernização da sociedade, especialmente nas capitais do país, levando as mulheres a buscarem um atendimento pré-natal, planejamento familiar e outros, realizando assim o exame preventivo da neoplasia cervical. Em 1974, foi criado o Centro Integrado de Controle do Câncer/CICAN em Salvador, a princípio, voltado exclusivamente para a atenção à saúde da mulher, e transformado em Serviço Estadual de Oncologia/CICAN em 1992, com maior abrangência. Este centro, desde a sua criação, vem oferecendo atendimento de prevenção e deteç̧ão do câncer cérvico-uterino, o que pode ter exercido influência no decréscimo desta mortalidade nesse município (BAHIA, 1996).

De acordo com alguns autores, o risco de morte por câncer cérvico-uterino é maior em idades elevadas, tendendo a um aumento progressivo com a idade, o que foi também verificado em Salvador. Este perfil também foi encontrado no México, Estados Unidos e Bélgica (ROBLES, 1996; VYSLOUZILOVA, 1997).

O impacto sócio-econômico da mortalidade por câncer cérvico-uterino nesse município foi analisado através do cálculo dos anos potenciais e anos produtivos de vida perdidos pois, segundo REICHENHEIM (1994), a morte em uma etapa produtiva da vida, penaliza o indivíduo e o grupo que lhe é próximo, além de privar a sociedade de seu potencial econômico e intelectual. Esta colocação aplica-se com mais propriedade ainda aos anos produtivos de vida perdidos, cujas médias encontradas por LESSA (1996), para as regiões Sudeste (14,4 anos) e Sul (16,0 anos), em 1988, são semelhantes àquela observada em Salvador $(15,4)$ neste mesmo ano. Por sua vez, a variação da média de 15,5 a 20,4 anos potenciais de vida perdidos por mulher, no período estudado, correspondeu a valores muito altos, especialmente por se tratar de uma causa de morte $100 \%$ evitável.

Assim, os resultados apontados por este estudo indicam que, embora tenha 
apresentado um declínio, ainda existe um grande risco de morte por câncer cervical em Salvador e, consequentemente, as perdas sociais são significativas e portanto, esta neoplasia continua a ser um grande problema de saúde pública nesta capital. Apesar do decréscimo observado, impõe-se uma maior atenção, por parte dos órgãos competentes, no desenvolvimento de atividades organizadas e continuadas de prevenção desta enfermidade e mortalidade. Ao descrever e analisar o padrão da tendência histórica desta causa de óbito em Salvador, o presente estudo poderá representar um subsídio para uma possível avaliação, nesta capital, do impacto do Programa Nacional de Combate ao Câncer de Colo Uterino realizado em 1998 pelo Ministério da Saúde no Brasil.

\section{AGRADECIMENTOS}

Os autores agradecem às professoras Maria da Conceição Nascimento Costa e Ines Lessa pelas críticas e sugestões apresentadas, e a todos aqueles que contribuiram para a realização deste trabalho.

\section{REFERÊNCIAS BIBLIOGRÁFICAS}

BAHIA. Secretaria de Saúde do Estado da Bahia (SESAB). Relatório Anual. Salvador: Serviço Estadual de Oncologia/CICAN, 1996.

BRASIL. Fundação Nacional de Saúde. Mortalidade Brasil - 1995. Brasília: CENEPI, 1998.

BRASIL Ministério da Saúde / Conselho Nacional de Saúde. Relatório Executivo da 11 a Reunião da Comissão Intersetorial de Saúde da Mulher. Brasília: CISMU, 1999.

BRASIL. Ministério da Saúde. Câncer no Brasil: dados dos Registros de Base Populacional. Rio de Janeiro: Instituto Nacional do Câncer /INCA, 1991.

BRASIL. Estimativa da incidência e mortalidade por câncer no Brasil. Rio de Janeiro: Instituto Nacional do Câncer /INCA, 1999.

BRASIL. Manual de orientação para 1994 - Dia Nacional do Combate ao Câncer. Rio de Janeiro: Instituto Nacional do Câncer /INCA, 1994.

BRASIL. O problema do câncer no Brasil. Rio de Janeiro: Instituto Nacional do Câncer / INCA, 1994. 
DIETZ, J. et al. Mortalidade por câncer de colo uterino no Rio Grande do Sul. Rev Ass Med Brasil, 39(3):146-50, 1993.

FAERSTEIN, E. Câncer cérvico-uterino e lesões precursoras: aspectos de ocorrência, controle e vigilância epidemiológica no Brasil. Rio de Janeiro, 1987. Dissertação de mestrado apresentada ao Instituto de Medicina Social da universidade do Estado do Rio de Janeiro.

FONSECA, L.A.M. Mortalidade por Câncer no Estado de São Paulo. Oncologia Atual, 2(1):6$14,1992$.

LAURENTI, R. et al. Estatísticas de Saúde. São Paulo: Ed. Pedagógica e Universitária Ltda., 1985.

LESSA, I. et al. Doenças crônicas não-transmissíveis no Brasil: dos fatores de risco ao impacto social. Bol Oficina Sanit Panam, 120(5):389-413, 1996.

MENDONÇA, G.A. e S. Câncer na população feminina brasileira. Rev. Saúde Pública, 27(1):68-75, 1993.

OPS. Manual de normas e procedimentos para o controle do câncer cérvico-uterino. Washington, 1985.

REICHENHEIM, M.E. Anos Potenciais de Vida Perdidos no Rio de Janeiro, 1990. As Mortes Violentas em Questão. Cad Saúde Pública, 10(supl. 1):188-98, 1994.

REVISTA BRASILEIRA DE CANCEROLOGIA. Rio de Janeiro: Instituto Nacional do Câncer IINCA, 42(4):207-8, 1996; 44(1):5-6, 1998 (Editoriais)

ROBLES, S.C. Tendencias de la mortalidad por cáncer de cuello de útero en las Américas. Bol Oficina Sanit Panam.121(6):478-90, 1996.

ROUQUAYROL, M.Z. Epidemiologia \& Saúde. Rio de Janeiro: MEDSI, 1994.

SPIEGEL, M. R. Estatística. 2ª ed., São Paulo: McGraw-Hill, 1985.

VYSLOUZILOVA, M.A et al. Cervical Cancer Mortality in Belgium, 1955-1989. A Descriptive Study. European Journal of Cancer, 33(11):1841-45, 1997. 


\title{
EVOLUTION OF THE MORTALITY FOR CERVICO-UTERINE CANCER IN SALVADOR - BA, 1979-1997
}

\begin{abstract}
SUMMARY: When early diagnosed and treated, cervical cancer is a perfectly avoidable cause of death. In Brazil, however, mortality from cervical cancer is still high and remains a public health problem. This study is aimed at both describing the evolution of such mortality and estimating the potential and productive years of life lost due to this neoplasia in Salvador (Ba), Brazil, between 1979 and 1997. Aggregates of time series were chosen for analysis by considering all deaths of women aged 20 or older who had either cervical cancer as their basic cause of death or cancer in an unspecified part of the cervix. Data from SUS/MS CD-ROM, IBGE and the Health Department of the State of Bahia/SESAB were used. Crude, age-specific and age-standardized mortality rates were taken as indicators. A decrease of $50,6 \%$ in the standardized mortality rate for this disease was noted in the period, with values ranging from $17,6 / 100.000$ women in 1979 to 8,7 in 1997. The risk of death due to this neoplasia was found to increase with age, markedly after the age of forty. The average of potencial years of life lost ranged from 15,5 in 1986 to 20,4 in 1980. Mortality from cervical cancer in Salvador was then shown to be still high and the possible determining factors for such evolution were discussed. The need for effective action on the prevention of such morbidity and mortality is emphasized.
\end{abstract}

KEYWORDS: cervical cancer; mortality; trends; neoplasia 


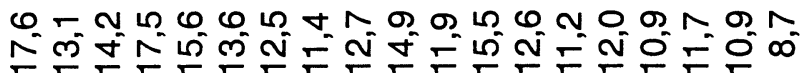

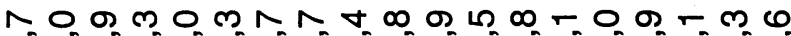
ก

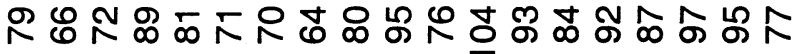

6 O-M กิ

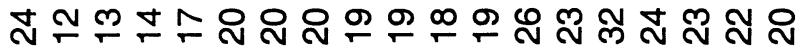

ก พ ठ ग ल

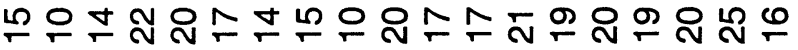

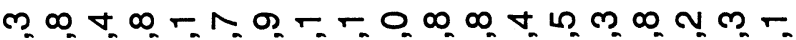
จ

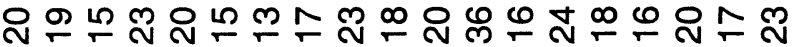

N ลं

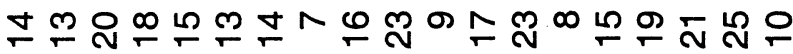

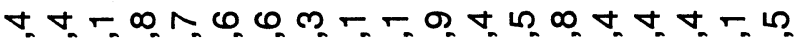

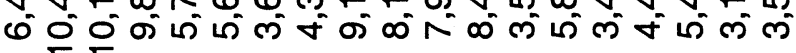

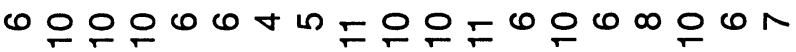

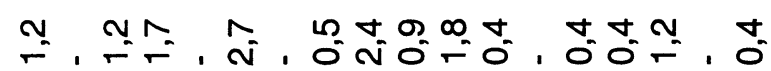

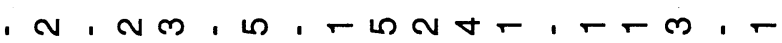

임ำ

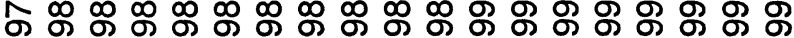




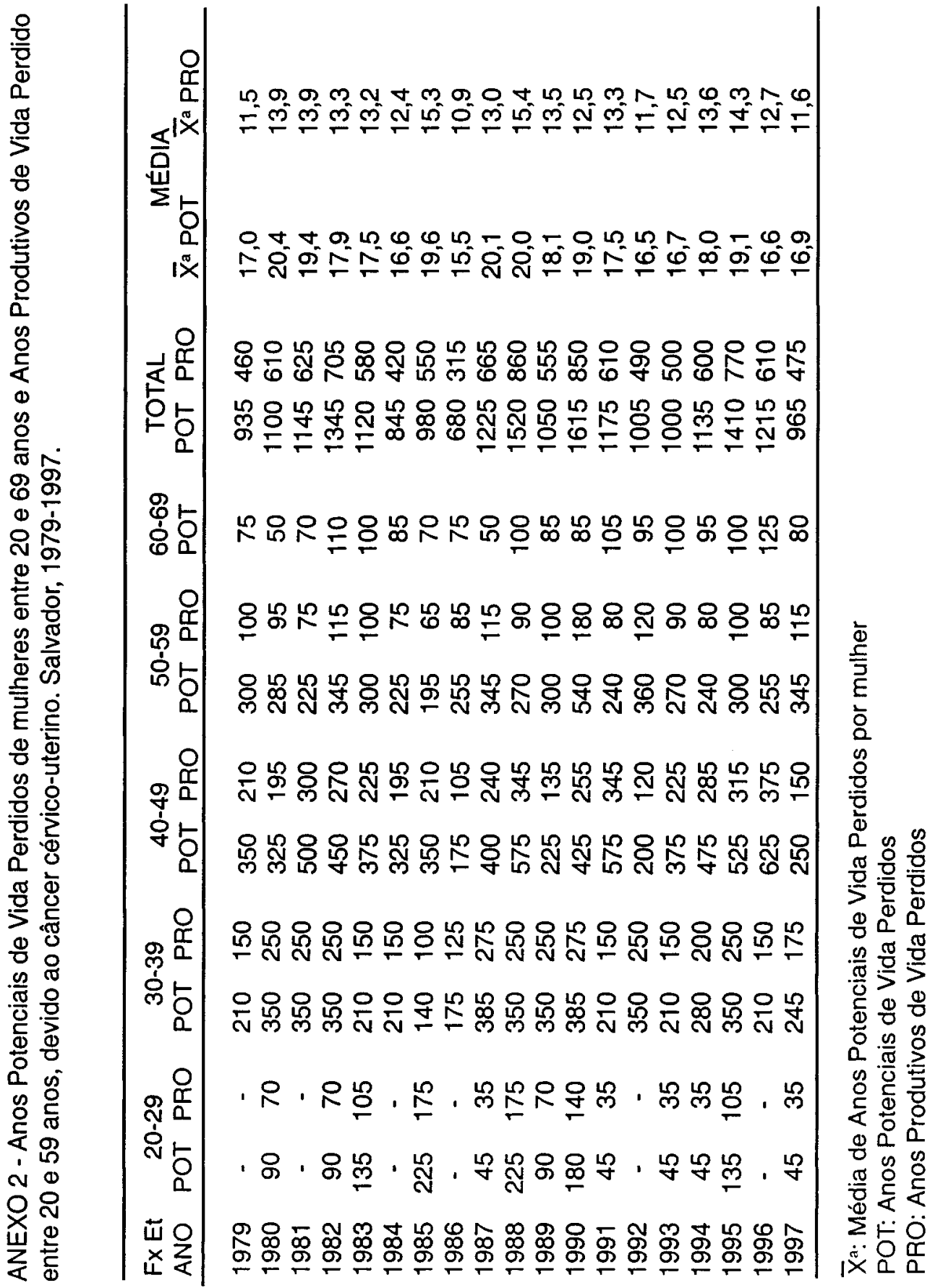

